\title{
A Generalised Approach on Generation of Commutative Matrix
}

\author{
Biswanath Rath \\ Department of Physics, North Orissa University, Takatpur, Baripada -757003, Odisha, INDIA \\ Email: biswanathrath10@gmail.com
}

\begin{abstract}
We proposed a generalised approach on generating commutative matrix of any nonsingular matrix $\mathrm{A}(\mathrm{NxN})$ satisfying the condition $\left[A, B_{i}\right]=0(\mathrm{i}=1,2,3,4, \ldots \ldots . \infty)$
\end{abstract}

Keywords: Inverse matrix, commutative matrix, continued fraction

Mathematical Classification (2010):16H15;14A05;05B20;15A8

\section{Introduction}

Matrix analysis is a powerful tool in understanding many feature of mathematics having direct relavance with physical systems. However it is commonly known that matrix multiplication has two important relations [1]

$$
\begin{gathered}
\operatorname{Det}(A B)=\operatorname{Det}(A) \operatorname{Det}(B)=\operatorname{Det}(B) \operatorname{Det}(A) \\
{[A, B] \neq 0}
\end{gathered}
$$

Interestingly it is possible to generate commutative matrices to a non-singular matrix[2,3]. In a recent paper any non-singular matrix $A(\mathrm{NxN})(\mathrm{N}=2,3)$ can possess commutative matrices $B_{L}$ provided

$$
B_{L}=\frac{1}{L+A}
$$

Mathematically

$$
\left[A, B_{L}\right]=0
$$

By varying $L$ one can generate infinite no of commutative matrices $B_{L}$. However in previous generation[3] ,the non-diagonal terms of entire $B_{L}$ remain invariant with that of $A$. Hence it is felt that one can generate new matrices having different diagonal and non-diagonal elements. The procedure is as follows.

\section{Commutative Matrices}

\subsection{Commutative Matrices: Series}

Here we suggest a procedure[2-4] to generate infinite matrices $B_{L}$ as Let $B_{L}$ is

$$
B_{L}=L+A+A^{2}+A^{3}+A^{4}+\ldots \ldots \ldots \ldots . . .=L+\sum_{k} A^{k}
$$

where $k=1,2,3,4, \ldots \ldots \infty$ Then it is easy to show that

$$
\left[A, B_{L}\right]=0
$$

and

$$
\left[B, B_{L}\right]=0
$$

From matrix theory [1] that one can have

$$
A, B_{L}, A^{-1}, B_{L}^{-1} \rightarrow \Psi
$$


Let

$$
A \Psi=\alpha \Psi
$$

and

$$
\begin{aligned}
A^{-1} \Psi & =\frac{1}{\alpha} \Psi \\
B_{L} \Psi & =\beta \Psi
\end{aligned}
$$

and

$$
B_{L}^{-1} \Psi=\frac{1}{\beta} \Psi
$$

Then

$$
\alpha|\Psi>=A| \Psi>
$$

multiplying both sides by $B_{L}^{-1}$ we have

$$
\alpha\left(B_{L}^{-1} \mid \Psi\right)>=\frac{\alpha}{\beta}\left|\Psi>=B_{L}^{-1} A\right| \Psi>
$$

Similarly

$$
B_{L}^{-1}\left|\Psi>=\frac{1}{\beta}\right| \Psi>
$$

Multiplying $A$ we have

$$
A B_{L}^{-1}\left|\Psi>=\frac{1}{\beta} A\right| \Psi>=\frac{\alpha}{\beta} \mid \Psi>
$$

Hence we have

$$
A B^{-1}=B_{L}^{-1} A \rightarrow \frac{\alpha}{\beta}
$$

In other words

$$
\left[A, B_{L}^{-1}\right]=0
$$

\subsection{Commutative Matrices: Continued Fraction}

Here we select $B$ as

$$
B_{F}=\frac{L}{L+\frac{A_{A}}{L+\frac{A_{A}}{L+\frac{A_{A}}{L+\frac{A_{1} \ldots \ldots}{L+A_{\ldots}}}}}}
$$

As in earlier ease it is easy to show that

$$
\left[A, B_{F}^{-1}\right]=0
$$

Hence we have two sets of commutative matrices $B_{L}^{-1}$ and $B_{F}^{-1}$. corresponding to $A$. Below we consider simple matrices and find out the form of $B_{L}$ and $B_{F}$ as follows.

\section{Infinite Generation of Commutative Matrices $\left(B_{L}\right)$}

\subsection{Infinite Generation of Commutative Matrices $\left(B_{L}\right)$ : Case Study $(2 \times 2)$}

Consider a simple (2x2) matrix $A$ as [1-4]

$$
A=\left[\begin{array}{ll}
2 & 1 \\
2 & 3
\end{array}\right]
$$

(i) $B_{1}=L+A$ as

$$
B_{1}=L+A=\left[\begin{array}{cc}
2+L & 1 \\
2 & 3+L
\end{array}\right]
$$


Let us consider different values of $L$ as follows $\mathbf{L}=\mathbf{1}$ In this case we get the known matrix [1] i.e

$$
B_{1}=\left[\begin{array}{ll}
3 & 1 \\
2 & 4
\end{array}\right]
$$

and

$$
B_{1}^{-1}=\left[\begin{array}{cc}
0.4 & -0.1 \\
-0.2 & 0.3
\end{array}\right]
$$

Then it easy to show that

$$
A B_{1}^{-1}=B^{-1} A=\left[\begin{array}{ll}
0.6 & 0.1 \\
0.2 & 0.7
\end{array}\right]
$$

(ii) $B_{2}=L+A+A^{2}(\mathbf{L}=\mathbf{1})$

In this case we get the known matrix [1] i.e

$$
B_{2}=\left[\begin{array}{cc}
9 & 6 \\
12 & 15
\end{array}\right]
$$

and

Then it easy to show that

$$
B_{2}^{-1}=\left[\begin{array}{cc}
\frac{5}{21} & \frac{-2}{21} \\
\frac{-4}{21} & \frac{1}{7}
\end{array}\right]
$$

$$
A B_{2}^{-1}=B_{2}^{-1} A=\left[\begin{array}{cc}
\frac{2}{7} & \frac{-1}{21} \\
\frac{-2}{21} & \frac{5}{21}
\end{array}\right]
$$

(iii) $B_{3}=L+A+A^{2}+A^{3}(\mathbf{L}=\mathbf{1})$

In this case we get the known matrix [1] i.e

$$
B_{3}=\left[\begin{array}{ll}
31 & 27 \\
54 & 58
\end{array}\right]
$$

and

Then it easy to show that

$$
B_{3}^{-1}=\left[\begin{array}{ll}
\frac{29}{170} & \frac{-27}{340} \\
\frac{127}{170} & \frac{31}{340}
\end{array}\right]
$$

$B_{4}=L+A+A^{2}+A^{3}+A^{4}(\mathbf{L}=\mathbf{1})$

$$
A B_{3}^{-1}=B_{3}^{-1} A=\left[\begin{array}{ll}
\frac{31}{170} & \frac{-23}{340} \\
\frac{-23}{170} & \frac{39}{340}
\end{array}\right]
$$

In this case we get the known matrix [1] i.e

$$
B_{4}=\left[\begin{array}{ll}
117 & 112 \\
224 & 229
\end{array}\right]
$$

and

Then it easy to show that

$$
B_{4}^{-1}=\left[\begin{array}{cc}
\frac{229}{1705} & \frac{-112}{1705} \\
\frac{1224}{1705} & \frac{117}{1705}
\end{array}\right]
$$

$$
A B_{4}^{-1}=B_{4}^{-1} A=\left[\begin{array}{cc}
\frac{234}{1705} & \frac{-107}{170.5} \\
\frac{--214}{1705} & \frac{127}{1705}
\end{array}\right]
$$

(v) $B_{5}=L+A+A^{2}+A^{3}+A^{4}+A^{5}(\mathbf{L}=\mathbf{1})$

In this case we get the known matrix [1] i.e

$$
B_{5}=\left[\begin{array}{ll}
459 & 453 \\
906 & 912
\end{array}\right]
$$

and

$$
B_{5}^{-1}=\left[\begin{array}{cc}
\frac{152}{1365} & \frac{-151}{2730} \\
\frac{-151}{1365} & \frac{51}{910}
\end{array}\right]
$$

Then it easy to show that

$$
A B_{5}^{-1}=B_{5}^{-1} A=\left[\begin{array}{cc}
\frac{51}{455} & \frac{-149}{2730} \\
\frac{-149}{1365} & \frac{157}{2730}
\end{array}\right]
$$




\subsection{Infinite Generation of Commutative Matrices $\left(B_{L}\right)$ : Case Study $(3 \times 3)$}

Here we just consider a simple example of (3x3) matrix[3] and generate suitable commutative counter part as follows. The explicit expression for $A$ is [3]

$$
A=\left[\begin{array}{ccc}
-2 & 1 & 2 \\
3 & -2 & 1 \\
-1 & 3 & 3
\end{array}\right]
$$

Considering the value of $\mathrm{L}=1$ we get $B_{5}=L+A+A^{2}+A^{3}+A^{4}+A^{5}$ as

$$
\begin{gathered}
B_{5}=\left[\begin{array}{ccc}
23 & 177 & 344 \\
341 & 73 & 227 \\
-77 & 491 & 858
\end{array}\right] \\
B_{5}^{-1}=\left[\begin{array}{ccc}
\frac{-227}{16388} & \frac{4259}{8811414} & \frac{38}{8895} \\
\frac{11363}{15504} & \frac{175}{13353} & \frac{883}{27785} \\
\frac{347}{7072} & \frac{-153}{21652} & \frac{-273}{14245}
\end{array}\right] \\
A B_{5}^{-1}=B_{5}^{-1} A=\left[\begin{array}{ccc}
\frac{163}{4300} & \frac{-331}{30967} & \frac{-64}{6375} \\
\frac{518}{2825} & \frac{-158}{8411} & \frac{-346}{5135} \\
\frac{2362}{3525} & \frac{418}{41459} & \frac{191}{4641}
\end{array}\right]
\end{gathered}
$$

\section{Infinite generation of Commutative Matrices $\left(B_{F}\right)$}

\subsection{Infinite Generation of Commutative Matrices $\left(B_{F}\right)$ : Case Study $(2 \times 2)$}

Consider a simple (2x2) matrix $A$ as [1-3]

$$
A=\left[\begin{array}{ll}
2 & 1 \\
2 & 3
\end{array}\right]
$$

Now consider the matrix (i) $B_{1}=L+A$ as

$$
B_{1}=L+A=\left[\begin{array}{cc}
2+L & 1 \\
2 & 3+L
\end{array}\right]
$$

Let us consider different values of $L$ as follows $\mathbf{L}=\mathbf{1}$ In this case we get the known matrix [1] i.e

$$
B_{1}=\left[\begin{array}{ll}
3 & 1 \\
2 & 4
\end{array}\right]
$$

and

$$
B_{1}^{-1}=\left[\begin{array}{ll}
3 & 1 \\
2 & 4
\end{array}\right]
$$

Then it easy to show that

$$
A B_{1}^{-1}=B^{-1} A=\left[\begin{array}{cc}
8 & 6 \\
12 & 14
\end{array}\right]
$$

Now consider the matrix

(ii) $B_{2}=L+\frac{A}{L+A}(\mathbf{L}=\mathbf{1})$

$$
\begin{aligned}
& B_{2}=\left[\begin{array}{ll}
\frac{17}{27} & \frac{-1}{27} \\
\frac{-2}{27} & \frac{16}{27}
\end{array}\right] \\
& B_{2}^{-1}=\left[\begin{array}{cc}
\frac{8}{5} & \frac{1}{5} \\
\frac{1}{5} & \frac{17}{10}
\end{array}\right]
\end{aligned}
$$


Then it easy to show that

$$
A B_{2}^{-1}=B_{2}^{-1} A=\left[\begin{array}{cc}
\frac{17}{5} & \frac{19}{10} \\
\frac{19}{5} & \frac{53}{10}
\end{array}\right]
$$

Now consider the matrix

(iii) $B_{3}=L+\frac{A}{L+\frac{A}{L+A}}(\mathbf{L}=\mathbf{1})$

$$
\begin{aligned}
& B_{3}=\left[\begin{array}{ll}
\frac{73}{145} & \frac{-14}{145} \\
\frac{128}{145} & \frac{59}{145}
\end{array}\right] \\
& B_{3}^{-1}=\left[\begin{array}{ll}
\frac{59}{27} & \frac{14}{27} \\
\frac{28}{27} & \frac{73}{27}
\end{array}\right]
\end{aligned}
$$

Then it easy to show that

$$
A B_{3}^{-1}=B_{3}^{-1} A=\left[\begin{array}{ll}
\frac{146}{27} & \frac{101}{27} \\
\frac{202}{27} & \frac{247}{27}
\end{array}\right]
$$

Now consider the matrix

(iv) $B_{4}=L+\frac{A}{L+\frac{A}{L+\frac{A}{L+A}}}(\mathbf{L}=\mathbf{1})$ as

$$
\begin{aligned}
& B_{4}=\left[\begin{array}{ll}
\frac{147}{260} & \frac{-31}{520} \\
\frac{231}{260} & \frac{263}{520}
\end{array}\right] \\
& B_{4}^{-1}=\left[\begin{array}{ll}
\frac{243}{145} & \frac{31}{145} \\
\frac{62}{145} & \frac{294}{145}
\end{array}\right]
\end{aligned}
$$

Then it easy to show that

$$
A B_{4}^{-1}=B_{4}^{-1} A=\left[\begin{array}{ll}
\frac{588}{145} & \frac{356}{145} \\
\frac{712}{145} & \frac{944}{145}
\end{array}\right]
$$

Now consider the matrix

$$
\begin{aligned}
B_{5}=L+\frac{A}{L+\frac{A}{L+\frac{A}{L+\frac{A}{L+A}}}}(\mathbf{L}=\mathbf{1}) \text { as } \\
B_{5}=\left[\begin{array}{ll}
\frac{1247}{2353} & \frac{-201}{2353} \\
\frac{-402}{2353} & \frac{521}{2353}
\end{array}\right] \\
B_{5}^{-1}=\left[\begin{array}{ll}
\frac{523}{260} & \frac{201}{520} \\
\frac{201}{260} & \frac{1247}{520}
\end{array}\right]
\end{aligned}
$$

Then it easy to show that

$$
A B_{5}^{-1}=B_{5}^{-1} A=\left[\begin{array}{ll}
\frac{1247}{260} & \frac{1649}{520} \\
\frac{1649}{260} & \frac{4143}{520}
\end{array}\right]
$$

\subsection{Infinite Generation of Commutative Matrices $\left(B_{F}\right)$ : Case Study $(3 \times 3)$}

Here we just consider a simple example of $(3 \times 3)$ matrix[3] and generate suitable commutative counter part as follows. The explicit expression for $A$ is [3]

$$
A=\left[\begin{array}{ccc}
-2 & 1 & 2 \\
3 & -2 & 1 \\
-1 & 3 & 3
\end{array}\right]
$$

Now consider the matrix $B_{5}=L+\frac{A}{L+\frac{A}{L+\frac{A}{L+\frac{A}{L+A}}}}(\mathrm{~L}=1)$ as

$$
B_{5}=\left[\begin{array}{ccc}
\frac{932}{1207} & \frac{-217}{4866} & \frac{-781}{5230} \\
-\frac{-1339}{1049} & \frac{427}{398} & \frac{325}{1269} \\
\frac{323}{500} & \frac{502}{1341} & \frac{4140}{16661}
\end{array}\right]
$$




$$
\begin{gathered}
B_{5}^{-1}=\left[\begin{array}{ccc}
\frac{549}{440} & \frac{279}{1210} & \frac{2479}{4840} \\
\frac{731}{440} & \frac{1201}{1210} & \frac{-119}{4840} \\
\frac{-163}{220} & \frac{542}{605} & \frac{895}{337}
\end{array}\right] \\
A B_{5}^{-1}=B_{5}^{-1} A=\left[\begin{array}{ccc}
\frac{-1019}{440} & \frac{2811}{1210} & \frac{2029}{467} \\
\frac{-141}{440} & \frac{-481}{1210} & \frac{1001}{236} \\
\frac{333}{220} & \frac{3288}{605} & \frac{2303}{312}
\end{array}\right]
\end{gathered}
$$

\section{Conclusion}

This paper is the modified and more generalised version of the previous work of Rath[3] in generating commutative matrix of any non-sinular matrix. In fact one can generate infinite set of commutative matrix. Further one can check that matrices generated in this paper are different from that generated in previous approach [3] using the known matrix $A$. Here for the benefit of reader we have constructed considered simple cases $(\mathrm{N} \times \mathrm{N})(\mathrm{N}=2,3)$. However one can take any positive value of $\mathrm{N}$.

Acknowledgments. Author is thankful to Referee for constructive remarks.

\section{References}

1. E.Kreyszig:Advanced Engineering Mathematics,Wiley india Pvt,Ltd(New Delhi ,India)(2011).

2. B.Rath:Int.Jour.Math.Trend.Tech. 59(3),171(2018).

3. B.Rath:Int.Jour.Math.Trend.Tech. 60(1),40(2018).

4. B.Rath:Int.Jour.Math.Trend.Tech. 60(3),191(2018). 